\title{
Manual-based treatment Part 2: the advantages of manual-based practice in psychotherapy
}

\begin{abstract}
Although treatment manuals have existed for many years, controversy surrounds their use and dissemination. In a previous article, we addressed assets and liabilities of using empirically supported treatments (ESTs) in psychotherapy, as well as obstacles to disseminating such treatments. In this article, we argue that using EST aids clinical work and is ethically sound. At the same time, we draw attention to problems that may arise for the field at large in implementing ESTs. Finally, we address reasons some clinicians are reluctant to use ESTs.
\end{abstract}

\section{Why ESTs should guide clinical practice}

We believe that there is an ethical imperative to provide treatments that work for our patients and that ESTs help us to do that. At the very least, providing helpful treatment entails following the Hippocratic oath to "first, do no harm." Beyond this basic point, though, practitioners sometimes disagree about what constitutes effective treatment. We frame success as a decrease in symptoms and distress, as well as a possible increase in functioning at work or in school and in relationships. Proponents of ESTs have drawn attention to the need for research that targets effectiveness (or how ESTs work in real clinical practice) as well as efficacy (ie, how ESTs work in controlled clinical trials with strict exclusion criteria and expertly trained therapists). Although effectiveness research is still fairly new, Chambless and Ollendick argue that the existing evidence suggests that ESTs are effective as well as efficacious. ${ }^{1}$ Thus, relying on efficacy studies is an acceptable way to identify treatments that work.

If the aim of psychotherapy is to help patients get better, ESTs offer some advantages over unstructured approaches. Perhaps the largest advantage of ESTs is that they take advantage of actuarial prediction. Actuarial prediction and judgement refers to making decisions based on large scale statistical data. Although clinicians tend to treat each case as individual, and many individual characteristics may seem to have relevance for treatment decision making, evidence based on large numbers of cases reveals that they often do not. For example, a person who presents with a history of childhood sexual abuse and is depressed may seem to require a focus on historical issues. Actuarial data suggest that simply knowing that the person is depressed means that he or she has a high probability of responding positively to short term manual-based treatment. A clinician may think that his or her patient is different from those in research studies and may therefore choose not to follow a research based treatment. Paradoxically, over the course of a clinician's career, this method of decision making is likely to produce poorer results than consistently relying on research based decision making. Thus, it is better to rely on a research based treatment that works for most people than to assume that each individual client is an exceptional case and won't be helped by a research based treatment.
Since ESTs take advantage of the superiority of actuarial prediction over clinical prediction, adherence to ESTs should lead to a higher percentage of successful outcomes for patients. ${ }^{2}$ In addition, actuarial or statistically based decision making is more accurate than clinical judgement. ${ }^{3}$ Therefore, clinicians who base their decisions on research are more likely to make better decisions than those who are guided by intuition alone. It is important to note that following a research based treatment does not mean that individual clinical decision making is abandoned; it means that the choice of treatment approach is based on available research. Within that approach, artistry, creativity, and judgement are ever present. ${ }^{4}$

Actuarial decision making offers other benefits as well. Wilson points out that the level of confidence a clinician has in his or her clinical judgements is unrelated to the accuracy of those judgements. ${ }^{5}$ This contradicts the training most therapists receive; we are taught to trust our judgement whenever in doubt. Even within the context of professional assessment, clinicians are taught to filter test results through the lens of "their best clinical judgement." The logic seems to be that if assessment tools like the Minnesota Multiphasic Personality Inventory can provide valuable information, such information can only be improved upon with clinical judgement. In fact, as Dawes points out, such ad hoc clinical judgements often detract from the accuracy of standardised test information. ${ }^{3}$ Wilson also notes that individualised clinical judgement is less reliable than standardised treatment because individualised treatments introduce errors and misguided strategies that are not present in manualised treatments. ${ }^{5}$ Thus, relying on clinical judgement does not guarantee better outcomes for patients and may make decisions less accurate.

Some literature suggests that structured treatments produce comparable or superior results to flexible treatments. ${ }^{6}{ }^{7}$ As such, using structured treatments may be preferable because they are a known entity. Another advantage of using ESTs is that supervisors are better able to track their supervisees when they use structured treatments. ${ }^{5}$ In addition, ESTs may allow patients to more easily identify and understand a given treatment, and, by extension, to make an informed decision about whether to partake of it.

\section{Overarching concerns about implementing ESTs}

Despite all the benefits of using ESTs, we believe there may be some problems with their implementation. It is important to note that these problems have nothing to do with ESTs per se, only with their administrative implementation. One concern is whether the rise and possible dominance of ESTs could lead the field to reduce the availability of other forms of therapy, thus limiting patients' choices for treatment. Or, could reliance on already existing ESTs lead the field away from developing new 
and innovative treatments that might be even more effective than current treatments? In a different vein, it is important to consider whether and how third party care providers will endorse ESTs: will they subsidise only the shortest of all ESTs, thereby limiting a patient's right to choose from various empirically supported treatments and limiting choices based on time rather than effectiveness? Although these concerns do not have direct implications for particular empirically supported manualbased treatments, the field needs to grapple with these issues in order to ensure the best care possible is provided to our patients and to honour and protect their rights to choose among various effective treatments. As Addis and Hatgis point out, ${ }^{8}$ it is important for researchers, clinicians, and administrators to be aware and respectful of the different values that guide each other.

\section{Therapists' concerns about implementing ESTs}

If we are to move toward using ESTs and manuals in clinical practice, then we must understand and address some of the concerns that therapists have about using ESTs. We will begin by listing some of the most prominent concerns about ESTs, and then we will suggest some strategies for addressing them.

Some therapists believe that ESTs are too simple to address the complexity of real clinical cases. Worse yet, some therapists believe that ESTs encroach on therapists' ability to be creative and autonomous in their treatment approaches and decisions. In addition, some are concerned that loyalty to a manual may require therapists to neglect important issues that arise in the course of therapy, but are not part of the manualised treatment. For example, a therapist using a manualised treatment for panic disorder may feel that she cannot address a patient's abusive relationship with her spouse. Other concerns revolve around the structured and directive nature of ESTs; some therapists fear that using ESTs would require them to engage in interventions that are inconsistent with their therapeutic orientation. Finally, some therapists resist using ESTs because they believe that the cases they encounter in clinical practice are more complicated and harder to work with than the examples given in training manuals.

A couple of themes emerge from the list of concerns above. The first is the idea that therapy is a creative and dynamic practice that cannot be reduced to a set of rules or guidelines. Indeed, many therapists prize the idea that therapy is an art form. Understandably, ESTs threaten this idea, but there are ways that therapists can use ESTs and still feel creative. Wilson contends that ESTs do not, and cannot, replace the therapeutic relationship. ${ }^{5}$ He goes on to argue that part of the "art" of therapy can be helping patients to engage in the change process, regardless of the modality of the treatment being provided. A strong therapeutic alliance is correlated with successful outcomes in therapy, and as such, it should serve as a base for all ESTs. As any seasoned therapist knows, nurturing and maintaining the therapeutic alliance requires skill and flexibility.

Another theme is the fear that therapists won't be able to attend to pressing therapeutic issues that seem unrelated to the treatment protocol. In fact, just as unstructured therapy requires flexibility, so too do ESTs. Addis et al argue that therapists can make creative use of the tensions between attending to protocol and addressing issues as they arise in therapy. ${ }^{9}$ They emphasise that the tensions therapists experience in delivering ESTs are not to be avoided, and can become integral parts of the therapeutic process. This issue aside, ESTs are often more flexible than most therapists realise. ${ }^{10}$ Greater familiarity with manuals and ESTs may alleviate some of the fear and concern about their use.

\section{Conclusions}

Finally, as Addis and Hatgis maintain, ${ }^{8}$ it is important for researchers, therapists, and administrators to communicate with each other and to approach the development and use of ESTs in a way that is respectful of all parties. ${ }^{8}$ Too often, hierarchical relationships result from the process of reforming mental health services. If we are to address therapists' concerns about ESTs, each member of the research/practice/administration triad must stand on equal footing with the others. Better communication and egalitarian relations might mitigate some of the tension and resistance therapists have to ESTs. In the end, it is the mandate to help those in need that should drive decision making. Ultimately, it will be increased communication and respect among all parties involved in providing therapeutic services that will allow the field to determine the role of ESTs in clinical practice.

\section{ABIGAIL K MANSFIELD, MA MICHAEL E ADDIS, PhD Clark University Worcester, Massachusetts, USA}

1 Chambless DL, Ollendick TH. Empirically supported psychological interventions: controversies and evidence. Annu Rev Psychol 2001;52:685-716.

2 Addis ME. Evaluating the treatment manual as a means of disseminating empirically validated psychotherapies. Clinical Psychology: Science and Practice 1997;4:1-11.

3 Dawes RM. House of cards: psychology and psychotherapy built on myth. New York: Free Press, 1994.

4 Addis ME, Wade WA, Hatgis C. Barriers to dissemination of evidencebased practice: addressing practitioners' concerns about manual-based psychotherapies. Clinical Psychology: Science and Practice 1999;6:430-41.

5 Wilson GT. Manual-based treatments: the clinical application of research findings. Behav Res Ther 1996;34:295-314.

6 Schulte D, Kunzel R, Pepping G, et al. Tailor-made versus standardized therapy of phobic patients. Advances in Behavior Research and Therapy 1992;14:67-92.

7 Jacobson NS, Schmaling KB, Holtzworth-Munroe A, et al. Researchstructured vs clinically flexibly versions of social learning-based marital therapy. Behav Res Ther 1989:27:173-80.

8 Addis ME, Hatgis C. Values, practices, and the utilization of empirical critiques in the clinical triad. Clinical Psychology: Science and Practice 2000;7:120-4.

9 Addis ME, Hatgis C, Soysa C, et al. The dialectics of manual-based psychotherapy. The Behavior Therapist 1999;22:130-2.

10 Heimberg RG. Manual-based treatment: an essential ingredient of clinical practice in the $21^{\text {st }}$ century. Clinical Psychology: Science and Practice 1998;5:387-90. 